\title{
Congelación de Semen Equino Bajo Dos Esquemas de Adición de Dimetilformamida
}

\author{
Freezing of Equine Semen Under Two Schemes of Addition of Dimethylformamide \\ Daniel Domingo Pérez Q. ${ }^{1}$, Mariano Acosta L. ${ }^{2}$, Giovanni Restrepo B. ${ }^{3}$, \\ Cesar Camacho ${ }^{4}$, Jair Pérez O. ${ }^{1,5}$
}

\section{Resumen}

El objetivo del estudio fue evaluar la calidad posdescongelación de semen equino sometido a dos esquemas de adición de dimetilformamida (DMF) durante la congelación. Se obtuvo el semen de cinco caballos criollos colombianos, el cual fue sometido a dilución en medio INRA $82^{\circledR}$ modificado con dos tratamientos de adición de DMF: T1, adición inmediata de 5\% de DMF; T2, adición fraccionada de 5\% de DMF (durante 10 min). El semen se empacó en pajillas de $0.5 \mathrm{ml}$ que se sometieron a congelación convencional. La movilidad espermática, la vitalidad y la morfología de los espermatozoides se evaluaron pre- y posdilución. Adicionalmente, se realizó un análisis posdescongelación de la movilidad y de la cinética espermática mediante un sistema computarizado, y la evaluación de la integridad de la membrana plasmática por la prueba hipoosmótica (HOS). Se observó una menor reducción de la movilidad espermática por la adición inmediata de DMF (T1) antes y después de la congelación, en comparación con la adición fraccionada del crioprotector $(\mathrm{T} 2)(\mathrm{p}<0.05)$. Se encontraron valores superiores de movilidad total y progresiva posdescongelación para T1, mientras el índice de rectitud (STR) fue mayor para $\mathrm{T} 2(\mathrm{p}<0.05)$. Se concluye que la adición inmediata de DMF durante la congelación, provee una mayor protección de la movilidad espermática posdescongelación del semen equino, respecto a la adición fraccionada de este crioprotector.

Palabras clave: diluyente; calidad seminal; criopreservación; crioprotector

\footnotetext{
${ }^{1}$ Facultad de Ciencias Agropecuarias, Universidad de La Salle, Bogotá, Colombia

${ }^{2}$ Facultad de Medicina Veterinaria y Zootecnia, Fundación Universitaria Autónoma de las Américas, Medellín, Colombia

${ }^{3}$ Facultad de Ciencias Agrarias, Universidad Nacional de Colombia, Medellín, Colombia

${ }^{4}$ Facultade de Ciências Veterinarias, Universidade Federal de Rio Grande do Sul, Porto Alegre, Brasil

${ }^{5}$ E-mail: jairperez@unisalle.edu.co
}

Recibido: 30 de marzo de 2017

Aceptado para publicación: 21 de julio de 2017 
The aim of this study was to evaluate the post-thawing quality of equine semen subjected to two dimethylformamide (DMF) addition schemes during freezing. Semen of five Colombian Creole horses was obtained and diluted in modified INRA82 ${ }^{\circledR}$ medium with two DMF addition treatments, T1: immediate addition of 5\% DMF, and T2: fractional addition of $5 \% \mathrm{DMF}$ (during $10 \mathrm{~min}$ ). Semen was packed into $05 . \mathrm{ml}$ straws and subjected to conventional freezing. Sperm motility, vitality and morphology before and after dilution were assessed. In addition, a post-thaw analysis of sperm motility and kinetics using a computerized system and the evaluation of plasma membrane integrity by the hypoosmotic test (HOS) were performed. Before and after freezing, a lower reduction in sperm motility was observed by the immediate addition of DMF (T1), compared to the fractional addition of the cryoprotectant $(\mathrm{T} 2)(\mathrm{p}<0.05)$. Superior values of total and progressive post-thaw motility for T1 were found, while the straightness index (STR) was higher for T2 $(p<0.05)$. It is concluded that the immediate addition of DMF during freezing provides a better protection of the post-thawing sperm motility of stallion semen than the fractional addition of this cryoprotectant.

Key words: extender; sperm quality; cryopreservation; crioprotectant

\section{INTRODUCCIÓN}

En los últimos años se han introducido en la industria equina mejoras a la criopreservación de espermatozoides, las cuales han facilitado el comercio nacional e internacional del semen equino. Este proceso, adicionalmente, ayuda al control de la diseminación de enfermedades venéreas $\mathrm{y}$, permite a su vez, la inseminación de varias yeguas con un solo eyaculado (Peña et al., 2011). No obstante, la calidad del semen descongelado es inferior respecto al semen fresco o refrigerado, lo cual queda demostrado por la disminución de la tasa de preñez (Hoffmann et al., 2011). Esto sucede por los cambios que sufre la célula espermática durante el proceso de congelación, que conllevan a la pérdida de la integridad y de la función de la membrana plasmática (Parks y Graham, 1992), atribuido al choque térmico, al estrés osmótico, al estrés oxidativo, a la formación de cristales de hielo y a la apoptosis (Peña et al., 2011).
En términos generales, la fertilidad con semen congelado en equinos es menor en comparación con la observada en otras especies. Este fenómeno se debe principalmente a factores propios del equino y a que la selección del reproductor se hace de acuerdo a su rendimiento en las diferentes disciplinas ecuestres, más no a su capacidad reproductiva (Clulow et al., 2007; Varner et al., 2015). Esto ha llevado a que los sementales sean catalogados como de buena o mala congelabilidad, con base en las características de su movilidad espermática posdescongelación (Vidament et al., 1997; Ortega-Ferrusola et al., 2009; Hoffmann et al., 2011).

Los procedimientos de criopreservación son conocidos por reducir la supervivencia y la habilidad fertilizante de los espermatozoides de los mamíferos, lo cual depende de la capacidad del semen para soportar los protocolos de congelación y descongelación, en base a las diferencias en la movilidad y la integridad de la membrana (Yeste et al., 2015). También se han hallado diferencias en la 
criotolerancia por efecto de la raza, para características como la integridad de la membrana plasmática y el potencial de membrana mitocondrial (Mascarenhas et al., 2012).

Se conoce gran cantidad de agentes crioprotectores que ejercen su función a nivel intracelular (penetrantes) o extracelular (no penetrantes) (Arifiantini et al., 2010). Entre los crioprotectores penetrantes, el glicerol es posiblemente el más utilizado para la congelación de semen equino; sin embargo, el uso de la dimetilformamida (DMF) ha proporcionado un aumento posdescongelación de la movilidad y de la integridad de la membrana plasmática de los espermatozoides, en comparación con el uso de glicerol (Vidament et al., 2001; Mesa y Henao, 2012).

Se conoce que la DMF, como parte del diluyente, reduce de forma significativa los efectos negativos de la congelación (Medeiros et al., 2002; Squires et al., 2004). Esto se atribuye a su menor peso molecular respecto a crioprotectores como el glicerol $\mathrm{y}$, por lo tanto, a su mayor potencial para atravesar la membrana plasmática; resultando en un equilibrio más rápido entre las regiones intra y extracelular y, teóricamente, en un menor potencial para generar daño espermático mediante el choque osmótico (Moffet et al., 2003).

La concentración del crioprotector ha sido un aspecto de interés en varios estudios. Medeiros et al. (2002) observaron una mejora en la movilidad posdescongelación del semen equino suplementado con $5 \%$ de DMF, respecto al uso de otros crioprotectores, incluyendo una mejora más notoria en equinos con semen de pobre congelabilidad. Un efecto similar fue observado por Hoffmann et al. (2011) quienes reportaron tasas de movilidad espermática superiores con el uso de concentraciones entre 2 y $4 \%$ de diferentes crioprotectores (incluyendo DMF), en caballos con pobre o con buena congelabilidad seminal. En otro estudio, se encontró una mayor viabilidad de semen equino congelado con $2 \%$ de DMF frente a concentraciones de 3 y $4 \%$ de DMF; igualmente, se observaron mejores resultados para la movilidad total (MT) y la fragmentación del ADN, cuando se empleó DMF en lugar de glicerol o la combinación de ambos crioprotectores (Gibb et al., 2013). De otro lado, Álvarez et al. (2014) reportaron que tanto el uso de $5 \%$ de DMF como la combinación $2.5 \%$ de DMF + $2.5 \%$ de glicerol adicionados al medio INRA96 ${ }^{\circledR}$, mejoraron la calidad posdescongelación del semen equino.

En vista a esto, se ha planteado la necesidad de evaluar no solo la concentración del crioprotector, sino también la influencia del esquema de adición del mismo antes de la congelación, sobre la integridad y la funcionalidad espermática posdescongelación. Esto, principalmente por la limitada tolerancia osmótica observada en el semen equino, que por efecto de crioprotectores como el glicerol, genera en los espermatozoides alteraciones de la movilidad, la viabilidad y la integridad del acrosoma (Ball y Vo, 2001). En este sentido, se ha encontrado que la adición paso a paso de DMF (en intervalos de un minuto) en condiciones de refrigeración a $5^{\circ} \mathrm{C}$, preservó la viabilidad espermática posdescongelación de forma superior a la adición de DMF en un solo paso (PérezOsorio et al., 2008).

Aunque los espermatozoides equinos son capaces de retornar a niveles normales de volumen celular después de ser incubados en soluciones anisosmolares (75-900 mosmol $/ \mathrm{kg}$ ), solo los cambios de osmolalidad media (sobre $450 \mathrm{mosmol} / \mathrm{kg}$ ) permiten que dichas células recuperen los niveles normales de función espermática (Pommer et al., 2002). Precisamente, teniendo en cuenta que la mayoría de los diluyentes de congelación son soluciones anisosmolares, la adición fraccionada de crioprotectores podría contribuir a disminuir el daño osmótico causado a los espermatozoides durante el sometimiento a los diluyentes. Por tanto, el objetivo de esta investigación fue evaluar la calidad posdescongelación de semen equino someti- 
do a dos esquemas de adición de DMF durante el proceso de congelación, con el fin de contribuir al mejoramiento de los protocolos de congelación de semen del caballo criollo colombiano y fortalecer las investigaciones de congelación de semen equino en Colombia.

\section{Materiales y Métodos}

\section{Equinos}

Se utilizaron cinco reproductores equinos criollos colombianos, previamente seleccionados mediante un examen clínico andrológico, con fertilidad comprobada y sexualmente activos. El rango de edad fue de 4 a 10 años. Los animales estuvieron ubicados en los municipios de Tabio y Madrid del departamento de Cundinamarca (Colombia) a $2650 \mathrm{msnm}$ y con una temperatura promedio de $16{ }^{\circ} \mathrm{C}$.

Los animales se mantuvieron en condiciones de confinamiento, bajo una dieta a base de heno angleton (Dichantun annulatum) a voluntad y concentrado en tres raciones diarias de $1.5 \mathrm{~kg} \mathrm{c} / \mathrm{u}$, así como agua a voluntad.

\section{Colecta y Evaluación Seminal}

Se realizó una serie de colectas diarias durante siete días para el agotamiento extragonadal de las reservas seminales, con el fin de mejorar la resistencia de los espermatozoides al proceso de criopreservación (Canisso et al., 2008).

La colecta del semen se realizó mediante una vagina artificial tipo Hannoverina a una temperatura de $52{ }^{\circ} \mathrm{C}$. En el momento de la colecta, se retiró mediante un filtro estéril la fracción en gel del eyaculado de la porción rica en espermatozoides (Varner, 2008).

Se realizó la evaluación de las características macroscópicas y microscópicas de cada eyaculado. El volumen se midió en una probeta previamente esterilizada, el $\mathrm{pH}$ se evaluó mediante una cinta indicadora $\left(\right.$ Merck $\left.^{\circledR}\right)$ y la concentración se calculó a través de un espectrofotómetro (Spermacue ${ }^{\circledR}$, Minitube) (Loomis y Graham, 2008). Mediante el uso de microscopía óptica convencional se evaluó la movilidad espermática total (porcentaje de células móviles) y progresiva (porcentaje de células móviles con trayectoria rectilínea), a partir de siete campos visuales y un aumento de 400X. Se determinó el vigor de los espermatozoides de forma subjetiva en una escala de 0 (ausente) a 5 (máximo) (Vidament et al., 2002). La morfología se evaluó mediante tinciones de eosina nigrosina, rojo congo, verde malaquita y azul de metileno. Asimismo, se realizó el conteo de 100 células, observando los defectos morfológicos de la cabeza, el acrosoma, la pieza intermedia y la pieza principal terminal (Brito, 2007).

\section{Procesamiento y Criopreservación del Semen}

El eyaculado se usó en proporción 1:1 con el diluyente Kenney ${ }^{\circledR}$ (Exodus Breeders Coorp, EEUU) sin crioprotector, temperado a $37.5^{\circ} \mathrm{C}$. El semen diluido se centrifugó a $600 \mathrm{~g}$ durante $12 \mathrm{~min}$ y se retiró el sobrenadante. El precipitado se trabajó con el diluyente INRA82 ${ }^{\circledR}$ (IMV Technologies, Francia) con $2 \%$ de yema de huevo, bajo los tratamientos: T1: adición inmediata de 5\% de DMF y T2: adición fraccionada de 5\% de DMF (durante $10 \mathrm{~min}$ ), según Pérez-Osorio et al. (2008). En ambos casos la dilución se realizó en cantidad suficiente para una concentración final de $200 \times 10^{6}$ espermatozoides $/ \mathrm{ml}$. Posterior a la dilución, la movilidad espermática fue evaluada mediante microscopía óptica para ambos tratamientos. El envasado de las pajillas se realizó de forma manual (Pérez-Osorio et al, 2008).

Las pajillas se sometieron a vapores de nitrógeno líquido por $15 \mathrm{~min}$ y a $6 \mathrm{~cm}$ de la superficie. Luego fueron sumergidas en nitrógeno líquido por $20 \mathrm{~s}$ y fueron almacenadas en un tanque criogénico durante 10 días 
Cuadro 1. Características del semen fresco de caballos criollos colombianos

\begin{tabular}{ccccccccc}
\hline Caballo & $\begin{array}{c}\text { Vol } \\
(\mathrm{ml})\end{array}$ & $\mathrm{pH}$ & Conc & $\begin{array}{c}\text { MT } \\
(\%)\end{array}$ & MP (\%) & Vigor & VE & MN \\
\hline 1 & 55 & 7 & 230 & 70 & 65 & 3 & 71 & 64 \\
2 & 32 & 7 & 264 & 75 & 70 & 3 & 76 & 74 \\
3 & 37 & 7 & 154 & 65 & 60 & 3 & 72 & 72 \\
4 & 15 & 7 & 340 & 80 & 75 & 3 & 73 & 70 \\
5 & 43 & 7 & 183 & 75 & 70 & 3 & 64 & 76 \\
\hline Media & $36.4 \pm$ & $7.0 \pm$ & $234.2 \pm$ & $73.0 \pm$ & $68.0 \pm$ & $3.0 \pm$ & $71.2 \pm$ & $71.2 \pm$ \\
\pm DE & 13.8 & 0.0 & 68.5 & 5.3 & 5.3 & 0.0 & 4.2 & 4.3 \\
\hline
\end{tabular}

DE: desviación estándar; Vol: volumen: Conc: concentración (x10 6 espermatozoides/ml); MT: movilidad total; MP: movilidad progresiva; VE: vitalidad espermática; MN: Morfología normal

hasta su descongelación (Medeiros et al., 2002).

Las muestras fueron descongeladas a $37.5{ }^{\circ} \mathrm{C}$ por $40 \mathrm{~s}$ en baño maría. Luego se realizó la evaluación posdescongelación de la movilidad, la morfología y la vitalidad espermática por los métodos previamente descritos. Adicionalmente, se realizó el análisis posdescongelación de la movilidad y la cinética espermática a través del método de análisis espermático asistido por computador (CASA), de acuerdo a lo descrito por Mesa y Henao (2012) y la evaluación de la integridad funcional de la membrana plasmática de acuerdo a lo reportado por Neild et al. (1999).

\section{Evaluación Estadística}

Se realizó un diseño de bloques completos al azar, donde cada bloque fue la unidad experimental. Se evaluó la normalidad de los datos mediante la prueba de ShapiroWills y las medias se compararon a través de la prueba de Tukey. Para las variables que no presentaron una distribución normal se realizó la prueba de Kruskal-Wallis para comparar las medianas. Todos los análisis se realizaron con el programa Statistix 8 (Analytical Software ${ }^{\circ}$, EEUU).

\section{Resultados y Discusión}

Las características seminales de los eyaculados colectados de caballos criollos colombianos se presentan en el Cuadro 1. En términos generales, estos resultados son similares a otros reportados para la raza (Restrepo et al., 2014, 2016).

El proceso de criopreservación expone las células al estrés inducido por las bajas temperaturas y por desequilibrios osmóticos. El estrés osmótico durante la criopreservación es predominantemente el resultado de la formación de hielo extracelular. Sin embargo, la adición de agentes crioprotectores también expone a las células al estrés osmótico (Sieme et al., 2016). La principal ventaja de crioprotectores como la DMF está en que causa menor daño osmótico a los espermatozoides que el glicerol, por su menor peso molecular y viscosidad (Alvarenga et al., 2016).

En esta investigación, la adición de DMF al semen diluido, de forma inmediata (T1) o fraccionada (T2) antes de la congelación, generó en ambos casos una reducción de la movilidad total (MT) y la movilidad progresiva (MP), que fue más severa para la 


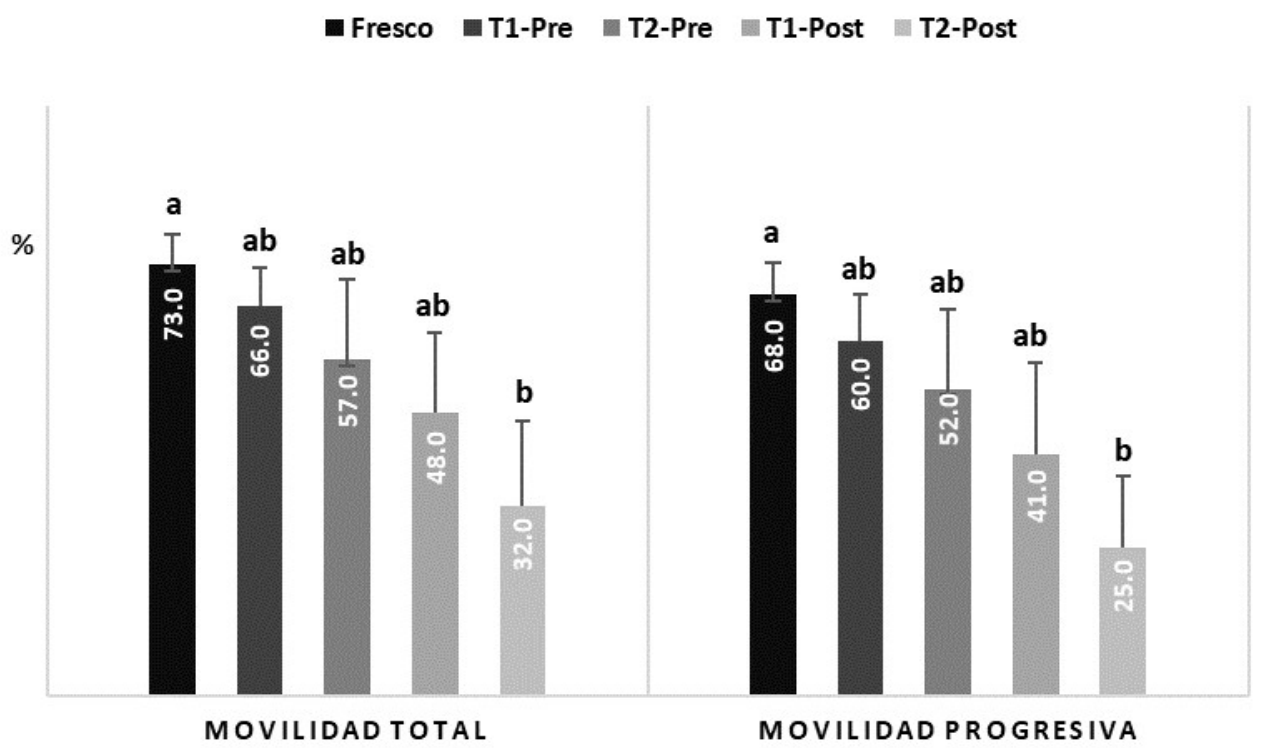

Figura 1. Efecto del esquema de adición de DMF sobre la movilidad pre y poscongelación. Letras diferentes indican diferencia estadística $(\mathrm{p}<0.05)$. T1-Pre: adición inmediata de DMF (precongelación). T2-Pre: adición fraccionada de DMF (precongelación). T1-Post: adición inmediata de DMF (poscongelación). T2Post: adición fraccionada de DMF (poscongelación)

adición fraccionada (Figura 1). A pesar de esto, no se encontró diferencia estadística para ambos parámetros entre el semen fresco y los tratamientos con DMF en la etapa de dilución del semen. Tampoco se encontró diferencia entre tratamientos con base a la movilidad espermática posterior a la dilución (Figura 1). Pérez-Osorio et al. (2008), en forma similar, tampoco encontraron diferencias en la movilidad espermática durante la etapa de dilución ni en la velocidad e integridad de la membrana de espermatozoides equinos sometidos a la adición inmediata o fraccionada de DMF.

Para el semen congelado, previamente sometido en ambos tratamientos a la adición de DMF, se observó una reducción de la calidad seminal posdescongelación. La movilidad espermática presentó una reducción significativa solo para la adición fraccionada de DMF (T2) respecto al semen fresco $(\mathrm{p}<0.05)$, mientras no se encontró diferencia entre $\mathrm{T} 1$ y T2, pese a la disparidad hallada entre las medias encontradas para MT y MP (Figura 1). Es posible que la alta variabilidad entre la congelabilidad del semen de los reproductores haya enmascarado el efecto posdescongelación del método de adición de DMF. En otros estudios se ha descrito la variabilidad existente en la calidad y criotolerancia del semen equino entre machos e incluso entre los eyaculados de un mismo ejemplar (Hoffman et al., 2011; Restrepo et al., 2014). A pesar de lo anterior, podría inferirse alguna influencia del esquema de adición de DMF en la criotolerancia de los espermatozoides equinos, la cual se hace evidente desde la dilución previa a la congelación y con un efecto derivado hacia la calidad seminal posdescongelación.

Los resultados del análisis computarizado de la movilidad y la cinética esper- 
Cuadro 2. Análisis de la movilidad y la cinética espermática posdescongelación en semen equino por efecto de la adición inmediata (T1) o fraccionada (T2) de 5\% de DMF

\begin{tabular}{lcc}
\hline Variable & T1 & T2 \\
\hline Movilidad total (\%) & $48.0 \pm 13.5^{\mathrm{a}}$ & $32.0 \pm 14.4^{\mathrm{b}}$ \\
Movilidad progresiva (\%) & $41.0 \pm 15.5^{\mathrm{a}}$ & $25.0 \pm 12.24^{\mathrm{b}}$ \\
Móviles progresivos rápidos (\%) & $10.5 \pm 2.1^{\mathrm{a}}$ & $13.3 \pm 10.8^{\mathrm{a}}$ \\
Velocidad curvilínea $(\mu \mathrm{m} / \mathrm{seg})$ & $74.9 \pm 4.2^{\mathrm{a}}$ & $76.1 \pm 6.7^{\mathrm{a}}$ \\
Velocidad lineal $(\mu \mathrm{m} / \mathrm{seg})$ & $25.7 \pm 2.6^{\mathrm{a}}$ & $26.8 \pm 5.5^{\mathrm{a}}$ \\
Velocidad media $(\mu \mathrm{m} / \mathrm{seg})$. & $43.8 \pm 2.1^{\mathrm{a}}$ & $40.2 \pm 7.6^{\mathrm{a}}$ \\
Índice de linealidad - LIN & $34.4 \pm 3.8^{\mathrm{a}}$ & $35.4 \pm 8.4^{\mathrm{a}}$ \\
Índice de rectitud - STR & $58.7 \pm 3.9^{\mathrm{b}}$ & $66.9 \pm 7.9^{\mathrm{a}}$ \\
Índice de oscilación - IO & $58.5 \pm 3.2^{\mathrm{a}}$ & $53.0 \pm 10.5^{\mathrm{a}}$ \\
Amplitud lateral de la cabeza ( $\mu \mathrm{m})$ & $3.2 \pm 0.3^{\mathrm{a}}$ & $3.0 \pm 0.4^{\mathrm{a}}$ \\
Frecuencia de batido (Hz) & $6.8 \pm 0.4^{\mathrm{a}}$ & $9.7 \pm 2.3^{\mathrm{a}}$ \\
Morfología normal $(\%)$ & $59.2 \pm 3.8^{\mathrm{a}}$ & $51.2 \pm 8.0^{\mathrm{a}}$ \\
Vitalidad espermática $(\%)$ & $52.8 \pm 7.8^{\mathrm{a}}$ & $53.6 \pm 13.9^{\mathrm{a}}$ \\
Integridad de la membrana plasmática - HOS $(\%)$ & $39.4 \pm 14.6^{\mathrm{a}}$ & $47.0 \pm 0.4^{\mathrm{a}}$ \\
\hline
\end{tabular}

$a, b$ Letras diferentes indican diferencia estadística significativa $(p<0.05)$

mática posdescongelación se presentan en el Cuadro 2. Se encontró diferencia significativa entre tratamientos para MT, MP y el Índice de rectitud (STR) $(\mathrm{p}<0.05)$, lo cual muestra un efecto del esquema de adición del crioprotector permeable DMF sobre los patrones de movimiento posdescongelación de los espermatozoides. Para los parámetros MT y MP se hallaron valores superiores cuando se realizó la adición inmediata de la DMF (T1), mientras que el STR fue mayor en el semen sometido a la adición fraccionada de DMF (T2). Otros parámetros donde se observó cierta tendencia por efecto de los tratamientos fueron la morfología normal con una media mayor para $\mathrm{T} 1$, mientras que la frecuencia de batido (BCF) y la integridad de la membrana plasmática (HOS) presentaron valores mayores para T2; sin embargo, en estos casos no se halló diferencia estadística (Cuadro 2).
Estos resultados difieren de los reportados por Mello (2005) y Pérez-Osorio et al. (2008), quienes mencionan una mejor preservación de la movilidad espermática, así como una mejora en la integridad del acrosoma y la integridad de la membrana plasmática con la adición fraccionada de DMF. Se indica que la adición de DMF en un solo paso causa una mayor alteración de la movilidad y la capacidad de la membrana para permitir la entrada y la retención de agua (Pérez-Osorio et al., 2008). Sin embargo, es posible que la propiedad de la DMF para reducir el tiempo de equilibrio antes de la criopreservación respecto a otros crioprotectores (Pukazhenthi et al., 2014), sumada a las condiciones particulares del protocolo de congelación, como la composición del diluyente y la curva de descenso de temperatura, pudiesen ser favorables para la adición inmediata de DMF; no siendo necesaria la adición fraccionada de este crioprotector. 
Adicionalmente, el análisis detallado de la movilidad realizado en este trabajo mediante un sistema CASA, podría proveer a diferencia de otros estudios mencionados, una valoración menos subjetiva de los resultados obtenidos. Es así que la evaluación de varios parámetros cinemáticos del semen pudo evidenciar las reducidas diferencias posdescongelación derivadas de los tratamientos de adición de DMF. Aunque son limitados los reportes de evaluación de los parámetros cinéticos del semen equino en relación con el efecto de la DMF y, aún menos, de su esquema de adición, se conoce que este crioprotector incrementa respecto al glicerol, el porcentaje de células rápidas y las velocidades lineal, curvilínea y media de los espermatozoides (Alvarenga et al., 2004).

Aún son pocas las investigaciones realizadas en congelación de semen del caballo criollo colombiano. Entre los trabajos reportados se encuentra la evaluación del uso de DMF y colesterol (Mesa y Henao, 2012); la evaluación de diferentes diluyentes de congelación (Restrepo et al., 2014); la evaluación de métodos de separación espermática y de la fertilización in vitro (Varela et al., 2015); y la relación entre la criotolerancia del semen y los componentes del plasma seminal (Usuga et al., 2016). Esta investigación contribuye al mejoramiento de los protocolos de congelación de semen del caballo criollo colombiano a través de la optimización del uso de la DMF como agente crioprotector.

\section{Conclusiones}

- La adición inmediata de dimetilformamida (DMF) durante la congelación del semen equino provee una mayor protección de la movilidad espermática posdescongelación, con respecto a la adición fraccionada del crioprotector.

- El esquema de adición de DMF al semen equino diluido no parece influir de forma importante en la vitalidad, la morfología y la integridad de membrana de los espermatozoides equinos sometidos a congelación.

\section{Literatura Citada}

1. Alvarenga $M$, Leão $K$, Papa $F$, Landim-Alvarenga $F$, Medeiros A, Gomes $\boldsymbol{G}$ 2004. The use of alternative cryoprotectors for freezing stallion semen. In: Proc Workshop on transporting gametes and embryos. Brewster, Massachusetts, USA. 74 p.

2. Alvarenga $M$, Papa $F$, Ramirez $C$. 2016. Advances in stallion semen cryopreservation. Vet Clin North Am Equine Pract 32: 521-530. doi: 10.1016/ j.cveq.2016.08.003

3. Álvarez C, Gil L, González N, Olaciregui M, Luño V. 2014. Equine sperm post-thaw evaluation after the addition of different cryoprotectants added to INRA 96® extender. Cryobiology 69: 144-148. doi: 10.1016/ j.cryobiol.2014.06.008

4. Arifiantini R, Purwantara B, Yusuf T, Sajuthi D. 2010. Effect of different cryoprotective agents on skim milk and dimitropoulus extender for stallion semen cryopreservation. J Indonesian Trop Anim Agric 35: 68-74. doi: 10.14710/ jitaa.35.1.68-74

5. Ball B, Vo A. 2001. Osmotic tolerance of equine spermatozoa and the effects of soluble cryoprotectants on equine sperm motility, viability, and mitochondrial membrane potential. J Androl 6: 10611069. doi: 10.1002/j.1939-4640.2001.tb03446.x

6. Brito L. 2007. Evaluation of stallion sperm morphology. Clin Tech Equine Pract 6: 249-264. doi: 10.1053/ j.ctep.2007.09.004

7. Canisso I, Souza F, Ortagiza Escobar J, Carvalho G, Morel M, da Silva E, et al. 2008. Congelamiento de semen de burro (Equus asinus). Rev Investig Vet Perú 19: 113-125. doi: 10.15381/ rivep.v19i2.1084 
8. Clulow J, Maxwell W, Evans G, Morris L. 2007. A comparison of duck and chicken egg yolk for cryopreser-vation of stallion sperm. Aust Vet J 85: 232-235. doi: 10.1111/j.1751-0813.2007.-00151.x

9. Gibb Z, Morris L, Maxwell W, Grupen C. 2013. Dimethyl formamide improves the postthaw characteristics of sexsorted and nonsorted stallion sperm. Theriogenology 79: 1027-1033. doi: 10.1016/j.theriogenology.2013.01.013

10. Hoffmann N, Oldenhof H, Morandini C, Rohn K, Sieme H. 2011. Optimal concentrations of cryoprotective agents for semen from stallions that are classified 'good' or 'poor' for freezing. Anim Reprod Sci 125: 112-118. doi: http:/ /dx.doi.org/10.1016/j.anireprosci.2011.03.001

11. Loomis P, Graham J. 2008. Commercial semen freezing: individual male variation in cryosurvival and the response of stallion sperm to customized freezing protocols. Anim Reprod Sci 105: 119-128. doi: 10.1016/j.anireprosci. 2007.11.010

12. Mascarenhas D, Martiliano D, Linhares Y, Ferreira S, Castelo M, Torres de Souza J. 2012. Avaliação dos diluidores Botu-crio ${ }^{\circledR}$ e Tris na criopreservação no sêmen de garanhões Quarto de Milha e Mangalarga Marchador. Ciência Anim Bras 15: 322-329. doi: 10.1590/1809-6891v15i325327

13. Medeiros A, Gomes G, Carmo, Papa F, Alvarenga M. 2002. Cryopreservation of stallion sperm using different amides. Theriogenology 58:273-276. doi: 10.1016/S0093-691X(02)-00898-1

14. Mello F. 2005. Avaliação in vitro da adição fracionada da dimetilformamida na criopreservação de sêmen eqüino. Dissertação Mestrado. Brasil: Univ Federal de Minas Gerais. 50 p.

15. Mesa A, Henao G. 2012. Efecto del colesterol y la dimetilformamida sobre parámetros posdescongelación en espermatozoides de caballos criollos colombianos. Revista MVZ Córdoba 17: 2908-2915.
16. Moffet P, Bruemmer J, Card C, Squires E. 2003. Comparison of dimethyl formamide and glycerol for cryopreservation of equine spermatozoa. In: Proc Society for Theriogenology Annual Conference. Columbus, OH, USA.

17. Neild D, Chaves G, Flores M, Mora N, Beconi M, Agiiero A. 1999. Hypoosmotic test in equine spermatozoa. Theriogenology 51: 721-727. doi: 10.1016/S0093-691X(99)00021-7

18. Ortega-Ferrusola C, Garcia B, Gallardo-Bolanos J, Gonzalez-Fernandez L, Rodriguez-Martinez, H, Tapia J, Peña F. 2009. Apoptotic markers can be used to forecast the freezeability of stallion spermatozoa. Anim Reprod Sci 114: 393-403. doi: 10.1016/j.anireprosci.2008.10.005

19. Parks J, Graham J. 1992. Effects of cryopreservation procedures on sperm membranes. Theriogenology 38: 209-222. doi: 10.1016/0093-691X(92)90231-F

20. Peña F, Macías García B, Samper J, Aparicio I, Tapia J, Ortega Ferrusola C. 2011. Dissecting the molecular damage to stallion spermatozoa: the way to improve current cryopreservation protocols? Theriogenology 76: 11771186. doi: $10.1016 / \mathrm{j}$.therioge-nology.2011.06.023

21. Pérez-Osorio J, Mello F, Juliani G, Lagares M, Lago L, Henry M. 2008. Effect on post-thaw viability of equine sperm using stepwise addition of dimethyl formamide and varying cooling and freezing procedures. Anim Reprod 5: 103-109.

22. Pommer A, Rutlant J, Meyers S. 2002. The role of osmotic resistance on equine spermatozoal function. Theriogenology 58: 1373-1384. doi: 10.1016/S0093-691X(02)01039-7

23. Pukazhenthi B, Johnson A, Guthrie H, Songsasen N, Padilla L, Wolfe B, Coutinho da Silva M, et al. 2014. Improved sperm cryosurvival in diluents containing amides versus glycerol in the Przewalski's horse (Equus ferus prze- 
walskii). Cryobiology 68: 205-214. doi: 10.1016/j.cryobiol.2014.01.013

24. Restrepo G, Cantero J, Montoya J. 2016. Efecto de la centrifugación sobre la integridad y la funcionalidad de espermatozoides equinos. Rev Bio Agro 14: 119-125. doi: 10.18684/BSAA(14)119125

25. Restrepo G, Usuga A, Montoya J, Celis A, Henao A. 2014. Evaluación de dos diluyentes para la criopreservación de semen de caballos de la raza criollo colombiano. Rev Lasallista Investig 11: 63-70.

26. Sieme H, Oldenhof H, Wolkers $W$. 2016. Mode of action of cryoprotectants for sperm preservation. Anim Reprod Sci 169: 2-5. doi: 10.1016/j.anireprosci.2016.02.004

27. Squires E, Keith S, Graham J. 2004. Evaluation of alternative cryoprotectants for preserving stallion spermatozoa. Theriogenology 62: 1056-1065. doi: 10.1016/j.theriogenology.2003.12.024

28. Usuga A, Restrepo G, Rojano B. 2016. Criotolerancia del semen equino, estabilidad oxidativa y componentes del plasma seminal. Rev Inv Vet Perú 27: 505517. doi: 10.15381/rivep.v27i3.12005

29. Varela E, Duque J, Ramirez M, Ocampo D, Montoya J, Restrepo G. 2015. Efecto de cuatro métodos de separación seminal sobre la calidad y la capacidad fertilizante in vitro de esperma-tozoides equinos criopreservados. Rev Inv Vet Perú 26: 451-461. doi: 10.15381/rivep.v26i3.11188

30. Varner D, Gibb Z, Aitken R. 2015. Stallion fertility: a focus on the spermatozoon. Equine Vet J 47: 16-24. doi: 10.1111/evj. 12308

31. Varner D. 2008. Developments in stallion semen evaluation. Theriogenology 70: 448-462. doi: 10.1016/ j.theriogenology.2008.04.023

32. Vidament M, Daire C, Yvon J, Doligez, P, Bruneau B, Magistrini M, Ecot P. 2002. Motility and fertility of stallion semen frozen with glycerol and/or dimethyl formamide. Theriogenology 58: 249-251. doi: 10.1016/S0093-691X(02)00854-3

33. Vidament M, Dupere A, Julienne P, Evain A, Noue P, Palmer E. 1997. Equine frozen semen: freezability and fertility field results. Theriogenology 48 : 907-917. doi: 10.1016/S0093-691X(97)00319-1

34. Yeste M, Estrada E, Rocha L, Marín H, Rodríguez-Gil J, Miro J. 2015. Cryotolerance of stallion spermatozoa is related to ROS production and mitochondrial membrane potential rather than to the integrity of sperm nucleus. Andrology 3:395-407. doi: 10.1111/andr.291 